\title{
EL CINE COMERCIAL COMO MEDIO DE APRENDIZAJE DE LA FARMACOLOGÍA
}

\section{Commercial cinema as a resource for learning Pharmacology}

\author{
Clara PÉREZ-MAÑÁ ${ }^{1,2}$; Magí FARRÉ1,2; Esther PAPASEIT ${ }^{1,2}$; Josep-Eladi BAÑOS³ \\ ${ }^{1}$ Facultad de Medicina, Universitat Autònoma de Barcelona, Barcelona (España). ${ }^{2}$ Servicio de Farmacología Clínica, \\ Hospital Universitari Germans Trias i Pujol-IGTP, Badalona (España). ${ }^{3}$ Facultad de Medicina, Universitat de Vic- \\ Universitat Central de Catalunya. Vic (España). \\ Correo electrónico: mfarre.germanstrias@gencat.cat
}

Fecha de recepción: 19 de enero de 2020

Fecha de aceptación: 11 de febrero de 2020

Fecha de publicación: 15 de diciembre de 2020

\begin{abstract}
Resumen
Las películas comerciales se utilizan cada vez más como método de enseñanza en muchas disciplinas universitarias. En medicina son un recurso importante para enseñar algunos aspectos médicos al ser ilustrados usando el lenguaje cinematográfico. La relación médico-paciente, los dilemas éticos o el profesionalismo son alguno de los temas más presentes en las películas comerciales. En el presente manuscrito tratamos del interés del cine comercial en el campo de la farmacología, cuyo conocimiento está en la frontera de los campos básicos y clínicos. Revisamos el uso de películas para mejorar la enseñanza y aprendizaje de la farmacología y la farmacología clínica. Por ello describimos algunas películas que son especialmente útiles para este propósito, como Awakenings / Despertares de 1990, Lorenzo's oil / El aceite de Lorenzo de 1992, The constant gardener / El jardinero fiel de 2005 y más recientemente Dallas Buyers Club (2013). Se incluyen algunas recomendaciones para su uso óptimo en la enseñanza de farmacología y farmacología clínica.

Palabras clave: películas comerciales; docencia; Farmacología; Farmacología Clínica; innovación en docencia.
\end{abstract}

Rev. Med. Cine. 2020; 16(4), 247-253 Ediciones Universidad de Salamanca / @®@ J. Med. Mov., 2020; 16(4), 247-253 


\title{
EL CINE COMERCIAL COMO MEDIO DE APRENDIZAJE DE LA FARMACOLOGÍA \\ CLARA PÉREZ-MAÑÁ; MAGÍ FARRÉ; ESTHER PAPASEIT; JOSEP-ELADI BAÑOS
}

\begin{abstract}
Commercial movies are increasingly used as a teaching method in many college disciplines. In medicine they are an important resource to teach some aspects of medicine that will gain a considerable benefit of being illustrated using the cinematographic language. Doctor-patient relationship, ethics dilemmas, or professionalism are the most considered in commercial movies. In the present paper we deal with the interest in the field of pharmacology, whose knowledge is in the frontier of basic and clinical fields. We review the use of movies for improving the teaching of pharmacology and clinical pharmacology. Some movies are especially useful to this purpose such as Awakenings (1990), Lorenzo's oil (1992), The constant gardener (2005) and more recently Dallas Buyers Club (2013). Some recommendations for its optimal use in the teaching of pharmacology and clinical pharmacology are included.
\end{abstract}

Key words: commercial movies; teaching; Pharmacology; Clinical Pharmacology; teaching innovation.

\section{Cine comercial en educación médica}

El uso de películas comerciales en educación y enseñanza universitaria tiene una larga tradición, pero el uso de películas para enseñar a estudiantes de medicina es relativamente reciente ${ }^{1}$. Desde el primer informe publicado sobre el uso del cine en la educación médica en $1979^{2}$, esta actividad ha recibido una atención creciente como consecuencia del interés del uso de las humanidades en la educación médica.

En las últimas décadas, se han publicado muchos artículos que describen el uso de películas populares o comerciales en la docencia de la medicina. En una revisión sistemática sobre el tema, Darbyshire y Baker ${ }^{3}$ enumeraron varias razones para usar el cine en la educación sanitaria. Las películas nos cuentan una historia de forma fácil y las tramas a menudo consideran temas que pueden iniciar fácilmente una discusión entre los alumnos. Los roles activos de los alumnos hacen de esta discusión un componente importante del aprendizaje constructivista. El cine utiliza la imagen y el sonido, que mejoran la capacidad de los alumnos para observar y escuchar. Además, las películas estimulan discusiones y reflexiones como aprendizaje activo. Esta implicación se considera que es comparable al aprendizaje experimentado durante las consultas reales con los pacientes.

El uso de películas comerciales con fines de enseñanza es relativamente común en muchos programas universitarios. Ha sido especialmente relevante en los campos de la medicina y otras ciencias de la salud. Los temas considerados incluyen la fisiología, la microbiología, la farmacología y la farmacología clínica, la bioética y ética médica, la relación médico-paciente, la investigación preclínica y clínica, la enfermedad mental, las adicciones, los cuidados paliativos, los efectos de la enfermedad en pacientes, el profesionalismo médico y los conflictos en la atención médica. Los médicos son personajes comunes en las películas y su representación puede ayudar a los estudiantes a conocer cómo el público en general ve la profesión médica ${ }^{3-10}$.

Cinema-educación se refiere al uso de películas en formatos electrónicos (DVD entre otros) o recursos en línea, particularmente clips de estos formatos de imagen, para educar a los estudiantes de medicina y los médicos residentes en los aspectos psicosociales de la medicina ${ }^{11}$. En los últimos años, las series de televisión sobre temas médicos también se están utilizando en este contexto de forma creciente ${ }^{7,12}$.

Rev. Med. Cine. 2020; 16(4), 247-253 Ediciones Universidad de Salamanca / @@ J. Med. Mov., 2020; 16(4), 247-253 


\section{EL CINE COMERCIAL COMO MEDIO DE APRENDIZAJE DE LA FARMACOLOGÍA \\ CLARA PÉREZ-MAÑÁ; MAGÍ FARRÉ; ESTHER PAPASEIT; JOSEP-ELADI BAÑOS}

Algunos artículos recientes en la literatura médica respaldan la idea de que el uso de tales formatos de imagen proporciona una forma innovadora y efectiva de enseñanza. Su relevancia se ha demostrado como se ha comentado en artículos originales, metaanálisis y la publicación de varios libros. También varias páginas albergan información sobre cine y educación médica, además hay algunos cursos universitarios presenciales y existe al menos una revista específica dedicada al tema (Revista de Medicina y Cine / Journal of Medicine and Movies) con más de quince años de vida ${ }^{13}$.

Las películas en medicina se pueden usar para conocer y aprender diferentes aspectos, entre ellos es relevante para la profesión el conocer cómo se describen en las películas la medicina y los médicos. Las películas o partes de estas (clips) se pueden usar de forma más concreta para enseñar varios aspectos específicos de la medicina.

Dependiendo de los autores, las películas se utilizan habitualmente como una herramienta adicional o complementaria. Así en algunas experiencias se usa una película completa para explicar algunos temas o seminarios, o se incluyen clips cortos de una película en algunas clases magistrales o seminarios.

Menos frecuente es que las películas sean la parte principal de una asignatura complementada con clases magistrales o que toda una asignatura se desarrolle mediante la visión, trabajo y comentarios de varias películas. En este caso todo el curso se basa en ver y discutir las películas. Esto último sólo es posible en la docencia de asignaturas con pocos créditos y con contenidos muy transversales, como por ejemplo la bioética general o cursos sobre la muerte digna.

En algunos casos, los docentes prefieren usar películas comerciales de cine (o más recientemente de plataformas), especialmente largometrajes que hayan sido éxitos de taquilla y/o de crítica. Otros profesores prefieren películas no comerciales (menos conocidas, películas locales) o documentales. La selección es relevante ya que es preferible que los alumnos no hayan visto la película o al menos recientemente, para facilitar su visión de nuevo para el curso ${ }^{1,14}$.

Las películas populares pueden ser muy útiles como herramientas de enseñanza. Sin embargo, para garantizar unos resultados adecuados se requieren algunas consideraciones. Baños y Bosch $^{1}$ recomendaron recientemente algunos principios que deben tenerse en cuenta. Es esencial leer la literatura sobre cada película y verla cuidadosamente para identificar los elementos educativos que van a ser empleados. Es importante verificar que el material sea apropiado para el conocimiento actual de los estudiantes. Las películas comerciales de duración estándar (aproximadamente 90 minutos) o de larga duración pueden ser difíciles de usar al precisar de mucho tiempo de visión. Usar escenas específicas (clips) es una buena opción en algunos casos. La plausibilidad de las situaciones o hechos presentados a veces puede ser más importante que su precisión objetiva. La discusión de la película con los alumnos debe limitarse a unas pocas cuestiones previamente identificadas por los docentes, con una formulación de objetivos claros y la preparación de preguntas para ser discutidas. El aspecto docente en las películas incluye tanto el conocimiento médico especifico como los elementos sociales y humanísticos de la medicina, que son también muy valiosos. Una evaluación adecuada del aprendizaje de los estudiantes después de la actividad es fundamental para establecer la legitimidad del uso de la película como actividad docente $^{1}$.

Cine comercial en la enseñanza de la Farmacología y la Farmacología Clínica

La Farmacología no es un tema fácil para incluir en las películas, ya que abarca muchos temas diferentes y la mayoría del conocimiento se basa en la memoria para recordar nombres, y acciones a menudo complejas. La primera experiencia del uso del cine en Farmacología Clínica fue publicada por Koren en 1993, en que describió la

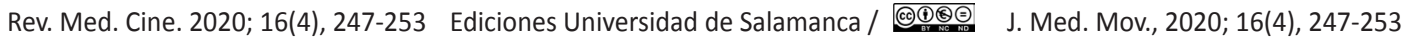




\section{EL CINE COMERCIAL COMO MEDIO DE APRENDIZAJE DE LA FARMACOLOGÍA \\ CLARA PÉREZ-MAÑÁ; MAGÍ FARRÉ; ESTHER PAPASEIT; JOSEP-ELADI BAÑOS}

utilización de la película comercial Awakenings / Despertares para explicar el uso de levodopa y las dificultades de la investigación clínica de nuevos tratamientos farmacológicos en Neurología ${ }^{15}$.

En la mayoría de las experiencias, el cine se usa sólo para explicar o apoyar algunos aspectos del uso de medicamentos, el uso inapropiado de los mismos, el abuso y la adicción a sustancias, o la introducción de los aspectos científicos y éticos de la investigación de nuevos medicamentos ${ }^{15-19}$.

En trabajos y revisiones recientes se describen aspectos específicos del uso del cine comercial en la enseñanza de la Farmacología y la Farmacología clínica ${ }^{14,20-22}$. Los objetivos del uso de películas para enseñar estas dos asignaturas se pueden encontrar en la tabla $1^{14,21,22}$. En las experiencias publicadas, la satisfacción de los estudiantes ha sido elevada y los resultados de aprendizaje adecuados ${ }^{21-22}$.

En nuestra experiencia hemos utilizado películas en la enseñanza de Farmacología / Farmacología Clínica en estudiantes de medicina y de biología

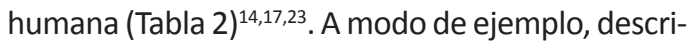
bimos en esta misma revista el uso de la película Dallas Buyers Club para conocer el proceso de desarrollo y aprobación de nuevos medicamentos $^{23}$. Para una mejor comprensión de la viabilidad de su uso, hemos incluido los objetivos educativos y los temas de discusión de esta película en las tablas 3 y 4 . A tenor de la actual pandemia SARSCoV-2, la película también permite reflexionar sobre las similitudes y diferencias de la pandemia de VIH-SIDA y SARS-CoV-2 en aspectos como la investigación epidemiológica, necesidad de tratamientos, investigación bajo presión y desarrollo de nuevos medicamentos. Más recientemente hemos explorado el uso de episodios de series de televisión en nuestras asignaturas de Farmacología y Farmacología Clínica ${ }^{24}$. La ventaja principal es que las series son muy populares y la duración de los episodios es más corta (aproximadamente 45-50 minutos), lo que facilita su programación. Un reciente artículo hace una relación de algunas de las series médicas que podrían ser objeto de evaluación para su uso docente ${ }^{25}$.
Tabla 1. Objetivos del uso de películas comerciales en Farmacología y Farmacología Clínica ${ }^{14,21,22}$.

1. Desarrollar los aspectos farmacológicos, terapéuticos, regulatorios y/o éticos más importantes de los temas seleccionados.

2. Conseguir que el estudiante comprenda los riesgos que representan los medicamentos y las novedades terapéuticas, así como su relevancia como causas prevenibles en la seguridad de los pacientes.

3. Conocer los retos científicos y éticos que surgen durante la investigación y el desarrollo de nuevos medicamentos.

4. Introducir al estudiante al análisis crítico de un problema, conseguir que razone sobre distintas posibles opciones, y que defienda oralmente y por escrito su opinión.

5. Reconocer en los personajes de ficción de las películas algunos signos y síntomas característicos de determinadas reacciones adversas a medicamentos o de determinadas enfermedades.

6. Concienciar a los estudiantes en los aspectos más humanos de la relación médico-enfermo, de la enfermedad y de su tratamiento.

Tabla 2. Algunas de las películas recomendadas por los autores en la enseñanza de la Farmacología y Farmacología Clínica.

Awakenings / Despertares (1990)

Lorenzo's oil / El aceite de la vida (1992)

The fugitive / El fugitive (1993)

Extreme measures / Al cruzar el límite (1996)

Miss Ever's boys / El experimento Tuskegee (1997)

The constant gardener / El jardinero fiel (2005)

Extraordinary measures / Medidas extraordinarias (2010)

Side effects / Efectos secundarios (2013)

Dallas Buyers Club (2013)

La fille de Brest / La doctora de Brest (2016)

En nuestra experiencia hemos utilizado mayoritariamente las películas en el contexto de seminarios. Previamente a los mismos o en el mismo momento de inicio, se entrega a los alumnos una descripción de los objetivos (ver Tabla 3 como ejemplo), se proyecta la película de 


\section{EL CINE COMERCIAL COMO MEDIO DE APRENDIZAJE DE LA FARMACOLOGÍA \\ CLARA PÉREZ-MAÑÁ; MAGÍ FARRÉ; ESTHER PAPASEIT; JOSEP-ELADI BAÑOS}

forma completa (muchas veces acompañada de un guion que incluye una serie de preguntas que deben completarse durante la visión para fomentar la atención). Una vez terminada la proyección se centran los temas de interés del debate (ver Tabla 4 como ejemplo), se discuten estos temas y se concluye el seminario. En algunos casos, unos días después, se debe entregar un resumen escrito muy breve sobre los aspectos que les han resultado más importantes y lo que se ha aprendido.

El uso de películas comerciales no está exento de algunas potenciales desventajas. A veces, las películas pueden no ser de interés para los estudiantes, pero en otros casos, su propio tema puede distraerlos del objetivo docente. Por parte de los docentes es necesaria una preparación cuidadosa, una moderación juiciosa de las discusiones y una evaluación crítica al finalizar. Las películas comerciales pueden aumentar la motivación de los estudiantes para comprender los principios de la Farmacología y Farmacología clínica y fomentar el aprendizaje activo.

\section{Tabla 3. Objetivos educativos de Dallas Buyers Club ${ }^{23}$.}

Entender el proceso del desarrollo de fármacos.

Comprender los principales aspectos implicados en el diseño de los ensayos clínicos como aleatorización, enmascaramiento, reclutamiento o consentimiento informado.

Roles esenciales en la investigación clínica: investigador, participantes y promotor/industria farmacéutica.

Debatir la problemática de los ensayos clínicos en enfermedades sin tratamientos eficaces.

Conocer las situaciones en que sería ético emplear placebo en ensayos clínicos.

Familiarizarse con el concepto de club de compradores y tráfico de fármacos ilegales o no aprobados. Uso de medicamentos fuera de sus indicaciones aprobadas.

Comprender el papel de las agencias gubernamentales que regulan los medicamentos para el uso humano (FDA, EMA y AEMPS).

Conocer los aspectos generales del sida, como los inicios de la epidemia, su curso clínico y el mecanismo de acción de los antirretrovirales.

\section{Tabla 4. Temas para el debate para Dallas Buyers Club ${ }^{23}$.}

Autonomía del paciente para escoger su tratamiento, especialmente en enfermedades graves:

Trafico de fármacos ilegales/no aprobados.

Relación con situaciones actuales (p.ej. cáncer).

Necesidad de un diseño experimental adecuado. Utilidad de la aleatorización y el enmascaramiento. Uso del placebo en ensayos clínicos.

Aprobación de fármacos: evaluación de la seguridad con estudios de poca duración frente a larga duración. Duración mínima de la investigación para conocer eficacia y/o seguridad:

Intereses de la industria farmacéutica, las agencias reguladoras y las asociaciones de pacientes.

Papel del médico clínico frente al papel del médico investigador y la problemática de que recaigan en la misma persona.

Similitudes y diferencias con otras pandemias como por ejemplo el HIV-SIDA.

\section{Referencias}

1. Baños JE, Bosch F. Using feature films as a teaching tool in medical schools. Educ Med. 2015;16 (4):206-11.

2. Fritz GK, Poe RO. The role of cinema in psychiatric education. Am J Psychiatry. 1979;136(2):207-10.

3. Darbyshire D, Baker P. A systematic review and thematic analysis of cinema in medical education. Med Humanit 2012;38(1):28-33.

4. Baños JE, Samsó E, Farré M. La fisiología en las películas: Coma, CO y la comprensión del intercambio gaseoso. Rev Med Cine. 2015;11(1):13-8.

5. Farré $\mathrm{M}$, Roset $\mathrm{PN}$, Bosch $\mathrm{F}$, Baños JE. Puting clinical pharmacology in context: the use of popular movies. J Clin Pharmacol. 2004;44(1):30-6.

6. Klemenc-Ketis Z, Kersnik J. Using movies to teach professionalism to medical students. BMC Med Educ. 2011; 11: 60.

7. Wong RY, Saber SS, Ma I, Roberts JM. Using television shows to teach communication skills in internal medicine residency. BMC Med Educ. 2009;9:9.

8. Alexander M, Hall MN, Pettice YJ. Cinemeducation: an innovative approach to teaching psychosocial medical care. Fam Med. 1994; 26(7): 430-3. 


\section{EL CINE COMERCIAL COMO MEDIO DE APRENDIZAJE DE LA FARMACOLOGÍA \\ CLARA PÉREZ-MAÑÁ; MAGÍ FARRÉ; ESTHER PAPASEIT; JOSEP-ELADI BAÑOS}

9. Farré M, Arribas S, Pérez J, Baños JE. Bioethical principles, clinical research and popular movies. Med Educ. 2013;47(11):1141-2.

10. Baños JE, Bosch F. Using feature films as a teaching aid with medical students. Med Teach. 2015;37(9):883-4.

11. Pérez J, Aramburu J, Baños JE, Bosch F, Díez J, Farré $\mathrm{M}$, et al. Uso del cine comercial como herramienta docente en estudios en ciencias de la salud. Una experiencia multidisciplinar y colectiva. Fundación Educación Médica. 2014;17(3):131-5.

12. Farré $A$, Torrens $M$, Baños JE, Farré M. CSI y la medicina forense. En: de la Torre T, coordinador. La medicina en las series de televisión. Barcelona: Fundación Dr. Antonio Esteve; 2016. p. 99-103. Cuadernos de la Fundación Dr. Antonio Esteve no 35.

13. García Moro M, García Sánchez JE, García Sánchez E, García Merino E. 15 años de la Revista de Medicina y Cine. Rev Med Cine [Internet]. 2019;15(1):1-2.

14. Farré $M$, Papaseit $E$, Pérez-Mañá $C$, Torrens $M$, Baños JE. Using popular movies in teaching: the case of pharmacology. In: Baños JE, Orefice C, editors. The role of humanities in the teaching of medical students. Barcelona: Fundació Dr. Antonio Esteve; 2018. p. 96103. Monographs of the Esteve Foundation № 38.

15. Koren G. Awakenings: using a popular movie to teach clinical pharmacology. Clin Pharmacol Ther. 1993;53(1):3-5.

16. Farré M, Arribas S, Pérez J, Baños JE. El uso de películas comerciales para comprender los principios bioéticos en la investigación clínica. Rev Med Cine [Internet]. 2013;9(4):151-5.

17. Farré $M$, Roset $P N$, Bosch $F$, Baños JE. Puting clinical pharmacology in context: the use of popular movies. J Clin Pharmacol. 2004;44(1):30-6.
18. Baños JE, Bosch F, Pérez J, Farré M. Al cruzar el límite / Extreme measures: cine, principios bioéticos e investigación clínica. Rev Med Cine [Internet]. 2011;7(3): 95-9.

19. Mateus JA, Baños JE, Papaseit E, Farré M. El lado oscuro de los fármacos en el cine. Rev Med Cine [Internet]. 2015;11(2):87-96.

20. Baños JE, Bosch F, Orefice C, Farré M. Del interés de las películas comerciales en la docencia de la Farmacología: veinticinco años de historia. Actualidad en Farmacología y Terapéutica (AFT). 2017;15 (4):313-7.

21. Ferrándiz ML, Noguera MA, Recio MC, Terencio MC, Montesinos MC, García- Arnandis I, et al. Farmacinema: recurso docente en el aprendizaje de Farmacología y Farmacia Clínica. Ars Pharm, 2010; 51 (supl 2):169-76.

22. Camarasa J, Escubedo E, Pubill D. Farmacocinema: aprender farmacología a través del cine. Edusfarm 2007;2:1-13.

23. De Haan-Bosch M, Gómez-Tomas A, Baños JE, Farré M. Dallas Buyers Club (2013): la investigación clínica con fármacos durante la epidemia del sida de los años 80. Rev Med Cine [Internet]. 2015;11(2):7381.

24. Baños JE, Lucena MI, Farré M, and the Group for the Study of the Teaching Effectiveness of TV series. The usefulness of TV medical dramas for teaching clinical pharmacology: A content analysis of House, M.D. Educ Med. 2019;20(5):295-303.

25. Cambra Badii I, Guardiola E, Baños JE. From Marcus Welby, M.D. to The resident: The changing portrayal of physicians in TV medical dramas. J Med Mov [Internet] [in press].

Rev. Med. Cine. 2020; 16(4), 247-253 Ediciones Universidad de Salamanca / @@@ J. Med. Mov., 2020; 16(4), $247-253$ 


Clara Pérez Mañá es médica especialista en Farmacología Clínica. Realizó
su formación MIR en el Hospital Vall d'Hebrón, después se trasladó al Insti-
tuto Hospital del Mar de Investigaciones Médicas-IMIM. En 2016 obtuvo
un contrato Juan Rodés. Desde 2017 es facultativa adjunta del Servicio
de Farmacología Clínica del Hospital Universitari Germans Trias i Pujol. Su
área de investigación es la neuropsicofarmacología clínica, en especial de
las sustancias de abuso. Además, ejerce labores docentes como profesora
asociada de Farmacología en la Facultad de Medicina de la Universidad
Autónoma de Barcelona (Unidad Docente Germans Trias).

